\title{
The Effect of Intravitreal Bevacizumab in Patients with Acute Central Serous Chorioretinopathy
}

\author{
Ji Won Lim, Su Jeong Ryu, Min Cheol Shin \\ Department of Ophthalmology, Chuncheon Sacred Heart Hospital, Hallym University College of Medicine, Chuncheon, Korea
}

Purpose: To evaluate the effect of intravitreal bevacizumab injection (IVBI) in acute central serous chorioretinopathy (CSC) patients.

Methods: Patients with acute CSC received IVBI $(1.25 \mathrm{mg} / 0.05 \mathrm{~mL})$ or observation by randomization. Twelve eyes in each group completed 6 months of regular follow-up and were ultimately included in this study. Each patient was assessed using best corrected visual acuity measurements, fluorescein angiography, and optical coherence tomography at baseline and had regular follow-ups after treatment.

Results: All patients showed improvements in visual acuity and fluorescein angiographic leakage and had resolution of their neurosensory detachment following treatment. There were no significant differences in visual acuity, central retinal thickness, or remission duration between the IVBI group and the control group at baseline or after treatment $(p>0.05)$.

Conclusions: Intravitreal bevacizumab showed no positive effect in acute CSC patients compared to the observation group, and there were no adverse effects of treatment. Further investigation will be helpful to understand this therapy in patients with CSC.

Key Words: Bevacizumab, Central serous chorioretinopathy, Randomized comparison, Therapeutics

Central serous chorioretinopathy (CSC) is characterized by the development of serous neurosensory retinal detachment at the posterior pole [1]. In the majority of patients, CSC is self-limited, and patients usually have a good visual prognosis. However, in some cases of CSC, patients develop progressive visual loss due to persistent serous retinal detachment, cystoid macular degeneration, or retinal pigment epithelium decompensation [2,3].

Clinicians usually elect to observe patients with acute CSC, because these patients generally show self-remission, and traditional treatments like laser photocoagulation or photodynamic therapy may induce complications [4,5]. However, patients with acute CSC often desire more expedient resolution of their disease.

The pathophysiology of CSC remains poorly understood. The advent of indocyanine green angiography, however, has

Received: September 8, 2009 Accepted: April 26, 2010

Reprint requests to Ji Won Lim. Department of Ophthalmology, Chuncheon Sacred Heart Hospital, Hallym University College of Medicine, \#153 Gyo-dong, Chunchon 200-704, Korea. Tel: 82-33-240-5176, Fax: 82-33255-5210, E-mail: jiwoneye@hallym.or.kr demonstrated choroidal involvement in the disease. It is likely that choroidal hyperpermeability is an early event in the development of symptomatic CSC where, under the appropriate circumstances, it may lead to retinal pigment epithelial detachment followed by neurosensory detachment [5]. Vascular endothelial growth factor (VEGF) has been implicated as the major factor responsible for increased vascular permeability [6]. Recently, bevacizumab (Avastin; Genentech Inc., San Francisco, CA, USA), an antibody to VEGF, has been shown to have anti-permeability properties. Intravitreal injection of bevacizumab (IVBI) has been reported to be associated with visual improvement and reduced neurosensory detachment without adverse events in patients with CSC [7]. In this study, we investigated the effect of IVBI in patients with acute CSC.

\section{Materials and Methods}

This was a prospective, randomized comparative study. Patients with symptomatic CSC of less than a 3-month duration were prospectively recruited between March 2008 and August 2008. The diagnosis of CSC was established by the presence of serous macular detachment on fundus examina- 
tion and dilated choroidal vasculature and hyperpermeability on indocyanine green angiography. Patients who had received any previous treatment, including photodynamic therapy or focal thermal laser photocoagulation for CSC, or who had evidence of choroidal neovascularization, polypoidal choriovasculopathy, or other maculopathy on clinical examination, fluorescein angiography, or indocyanine green angiography were excluded from the study. Informed consent was obtained from all subjects. The protocol was approved by the Institutional Review Board of the hospital. Patients were randomized into the IVBI group or the observation group at a ratio of $1: 1$. The randomization sequence was generated using a computerized randomization table.

Patients in the IVBI group received only a single intravitreal injection of bevacizumab $(1.25 \mathrm{mg}$ in $0.05 \mathrm{~mL})$ under standard protocol conditions. Eyes were injected less than one week after diagnosis in our clinic. The observation group was observed without any treatment or any medication. Each patient underwent clinical assessments, including best-corrected visual acuity measurement in Snellen units, applanation tonometry, fundus examination, fluorescein angiography, indocyanine green angiography, and optical coherence tomography (OCT) at baseline. Baseline central retinal characteristics were analyzed using OCT (Stratus III OCT ver. 4.0; Carl Zeiss Meditec, Dublin, CA, USA) with 6 diagonal, slow 6-mm radial line scans through a dilated pupil. The central 1-mm macular thickness (CMT) was obtained using the macular thickness map for our calculations.

Regarding follow-up, the patients were examined at 4-week intervals with slit-lamp biomicroscopy and OCT, and fluorescein angiography was performed at the discretion of the examiner. No other treatment for CSC was performed during the study. The primary outcome of the study was the time measured from baseline to complete absorption of subretinal fluid during follow-up. Secondary outcome measures included serial changes in the logarithm of the minimum angle of resolution $(\log M A R)$ visual acuity and OCT CMT.

Statistical analyses were performed using a commercially available statistical software package (SPSS ver. 11.5; SPSS
Inc., Chicago, IL, USA). Snellen units were converted to $\log$ MAR units for statistical analysis. Univariate categorical analyses were performed using the Mann-Whitney $U$-test or the Fisher exact test as appropriate. Data was analyzed via repeated-measures analysis of variance with Bonferroni correction. Statistical significance was set at 0.05 (2-sided) in all tests.

\section{Results}

We collected data from 32 eyes during the study period. Four eyes were excluded due to irregular follow-up, and four eyes were excluded due to the lack of post-IVBI OCT data. Thus, 24 eyes with at least 6 months of follow-up were ultimately included in this study. The mean age \pm SD of the patients was $43.2 \pm 9.0$ years. There were 20 men and 4 women. The IVBI group included 12 patients (12 eyes), and the observation group included 12 patients (12 eyes). No ocular or systemic complications were detected during the follow-up period after IVBI. Demographic data on sex, age, laterality of the eyes, and symptom duration showed no significant differences between the 2 groups. The differences in baseline visual acuity (logMAR) and baseline CMT between the 2 groups were not significant. The baseline characteristics of the 2 groups are shown in Table 1.

All patients in the 2 groups had complete resolution of their macular subretinal fluid during the 6 months of follow-up. The mean remission periods from diagnosis were $13.4 \pm 4.8$ weeks in the observation group and $13.6 \pm 7.6$ weeks in the IVBI group. There was no statistically significant difference between the 2 groups ( $p=0.783$, Mann-Whitney $U$-test).

After 6 months of follow-up, the mean \pm SD visual acuity of the IVBI group improved to $0.02 \pm 0.04$, whereas that of the observation group improved to $0.02 \pm 0.05$. The difference between the 2 groups was not statistically significant $(p=0.287$, Mann-Whitney $U$-test). The mean logMAR visual acuity of the 2 groups showed no difference at any follow-up visit. After 6 months of follow-up, the means \pm SD CMT for the

Table 1. Baseline characteristics of patients with acute central serous chorioretinopathy

\begin{tabular}{lccc}
\hline \multicolumn{1}{c}{ Characteristics } & $\begin{array}{c}\text { Observation } \\
(\mathrm{n}=12)\end{array}$ & $\begin{array}{c}\text { Intravitreal bevacizumab } \\
(\mathrm{n}=12)\end{array}$ & $p$-value \\
\hline Age (yr) & $40.7 \pm 7.0$ & $45.6 \pm 10.4$ & $0.234^{*}$ \\
Gender (male:female) & $11: 1$ & $9: 3$ & $0.217^{\dagger}$ \\
Laterality (right:left) & $8: 4$ & $5: 7$ & $0.272^{\dagger}$ \\
Number of CSC episodes (first:second) & $9: 3$ & $7: 5$ & $0.894^{\dagger}$ \\
Duration of current CSC episode (wk) & $5.4 \pm 1.6$ & $5.8 \pm 1.5$ & $0.789^{*}$ \\
Spherical equivalent refractive error (diopters) & $-0.50 \pm 1.2$ & $-0.33 \pm 1.2$ & $0.567^{*}$ \\
Baseline logMAR BCVA & $0.20 \pm 0.21$ & $0.23 \pm 0.21$ & $0.721^{*}$ \\
Baseline central macular thickness $(\mu \mathrm{m})$ & $442 \pm 160$ & $431 \pm 107$ & $0.977^{*}$ \\
\hline
\end{tabular}

Values are presented as mean $\pm \mathrm{SD}$ unless otherwise indicate. $\mathrm{SD}=$ standard deviation; $\mathrm{CSC}=$ central serous chorioretinopathy; logMAR $\mathrm{BCVA}=$ logarithm of minimal angle of resolution best-corrected visual acuity.

"Mann-Whitney $U$-test; ${ }^{\dagger}$ Fisher exact test. 

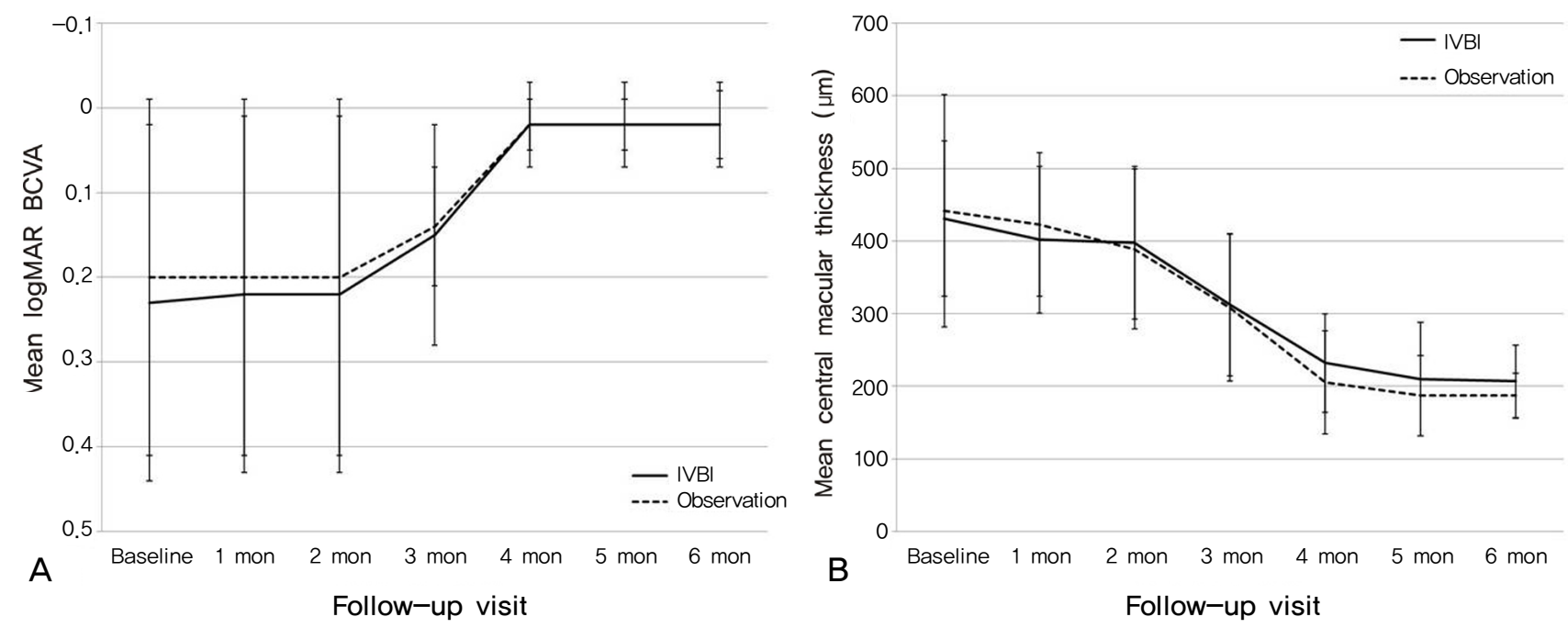

Fig.1. (A) Graph showing serial changes in the mean logarithm of the minimum angle of resolution best-corrected visual acuity (logMAR BCVA) for patients in the intravitreal bevacizumab injection (IVBI) group (solid line) and in the observation group (dashed line). Error bars represent standard errors of the mean. (B) Graph showing the serial changes in mean central macular thicknesses as measured using optical coherence tomography in the IVBI group (solid line) and in the observation group (dashed line). Error bars represent standard errors of the mean.

IVBI group and the control group were $207 \pm 50 \mu \mathrm{m}$ and $187 \pm 31 \mu \mathrm{m}$, respectively ( $p=0.377$, Mann-Whitney $U$-test $)$. The mean CMTs of the 2 groups showed no difference at any visit during the follow-up period. The serial changes in visual acuity and CMT are shown in Fig. 1.

\section{Discussion}

Typical acute CSC is characterized by duration of symptoms and/or retinal detachment of less than 6 months and monofocal or paucifocal fluorescein angiographic retinal pigment epithelium leakage [5,8]. The treatment of CSC is based largely on observation. The high spontaneous remission rate favors conservative management, lifestyle counseling, and discontinuation of glucocorticoid medication as first-line therapeutic options. Such a strategy can be expected to be followed by a resolution of detachment in nearly $90 \%$ of cases within 1.5 months $[9,10]$. If detachment persists for greater than 6 months, photocoagulation or photodynamic therapy should be considered. Although in most cases visual acuity returns to $20 / 25$ or better, patients often desire earlier recovery of visual acuity and faster disease remission. Photocoagulation may induce scotoma formation in the central visual field through retinal pigment epithelium damage at the macula, and photodynamic therapy necessitates the avoidance of physical activity after treatment and is associated with high cost in South Korea. The relatively good visual acuity of CSC patients and clinician concern about the possibility of complications gives cause to avoid treatment in the acute period. However, intravitreal injection can be conducted in the office and does not necessitate limitation of activity. Rarely, intravitreal injection is associated with complications like endophthalmitis or intraocular inflammation. We con- sider intravitreal injection to be a new treatment option in acute CSC patients and an alternative to observation or another treatment.

Vascular endothelial growth factor has profound effects on vascular permeability. Bevacizumab is a full-length antibody that binds all isoforms of VEGF. A growing number of reports in the literature support its safety and efficacy in many disorders [11-14]. However, the direct role of VEGF in CSC remains unknown. Indocyanine green angiography in patients with CSC has demonstrated evidence of choroidal lobular ischemia, choroidal venous congestion, and multiple areas of choroidal vascular hyperpermeability $[15,16]$. Choroidal ischemia in CSC may induce an increase in the concentration of VEGF. Thus, bevacizumab may theoretically reduce choroidal hyperpermeability in CSC. Although there have been no reports measuring VEGF levels in CSC, there are 2 reports of treatment with IVBI in patients with acute CSC. Torres-Soriano et al. [7] reported the use of IVBI in 5 eyes and found that the procedure was associated with visual improvement without adverse events. Seong et al. [17] also reported that 10 eyes showed resolution of neurosensory detachment within 1 month after IVBI. That study, however, was a small case series and had no control group.

Our results demonstrate that, in patients with acute CSC, IVBI showed no positive or negative effect in terms of earlier remission, functional results, or anatomical results. No adverse events were found to be associated with the treatment. Limitations of this study include the small number of patients and the short follow-up period. Also, there are various forms of CSC, and only acute CSC was investigated in this study. We did not investigate recurrence or long-term effects. Furthermore, there is no data available to support or refute the proposed mechanism of IVBI in CSC. Further inves- 
tigation will be helpful for understanding this therapy in patients with CSC.

\section{Conflict of Interest}

No potential conflict of interest relevant to this article was reported.

\section{References}

1. Gass JD. Pathogenesis of disciform detachment of neuroepithelium: II. Idiopathic central serous choroidopathy. Am J Ophthalmol 1967;63:587-615.

2. Levine R, Brucker AJ, Robinson F. Long-term follow-up of idiopathic central serous chorioretinopathy by fluorescein angiography. Ophthalmology 1989;96:854-9.

3. Loo RH, Scott IU, Flynn HW Jr, et al. Factors associated with reduced visual acuity during long-term follow-up of patients with idiopathic central serous chorioretinopathy. Retina 2002;22:19-24.

4. Chan WM, Lai TY, Lai RY, et al. Half-dose verteporfin photodynamic therapy for acute central serous chorioretinopathy: one-year results of a randomized controlled trial. Ophthalmology 2008;115:1756-65.

5. Wang M, Munch IC, Hasler PW, et al. Central serous chorioretinopathy. Acta Ophthalmol 2008;86:126-45.

6. Tolentino MJ, McLeod DS, Taomoto M, et al. Pathologic features of vascular endothelial growth factor-induced retinopathy in the nonhuman primate. Am J Ophthalmol 2002;133: 373-85.

7. Torres-Soriano ME, Garcia-Aguirre G, Kon-Jara V, et al. A pi- lot study of intravitreal bevacizumab for the treatment of central serous chorioretinopathy (case reports). Graefes Arch Clin Exp Ophthalmol 2008;246:1235-9.

8. Eandi CM, Ober M, Iranmanesh R, et al. Acute central serous chorioretinopathy and fundus autofluorescence. Retina 2005; 25:989-93.

9. Wong R, Chopdar A, Brown M. Five to 15 year follow-up of resolved idiopathic central serous chorioretinopathy. Eye 2004:18:262-8.

10. Sharma T, Shah N, Rao M, et al. Visual outcome after discontinuation of corticosteroids in atypical severe central serous chorioretinopathy. Ophthalmology 2004;111:1708-14.

11. Avery RL, Pieramici DJ, Rabena MD, et al. Intravitreal bevacizumab (Avastin) for neovascular age-related macular degeneration. Ophthalmology 2006;113:363-72.

12. Spaide RF, Laud K, Fine HF, et al. Intravitreal bevacizumab treatment of choroidal neovascularization secondary to age-related macular degeneration. Retina 2006;26:383-90.

13. Heiduschka P, Fietz H, Hofmeister S, et al. Penetration of bevacizumab through the retina after intravitreal injection in the monkey. Invest Ophthalmol Vis Sci 2007;48:2814-23.

14. Shahar J, Avery RL, Heilweil G, et al. Electrophysiologic and retinal penetration studies following intravitreal injection of bevacizumab (Avastin). Retina 2006;26:262-9.

15. Stanga PE, Lim JI, Hamilton P. Indocyanine green angiography in chorioretinal diseases: indications and interpretation: an evidence-based update. Ophthalmology 2003;110:15-21.

16. Piccolino FC, Borgia L. Central serous chorioretinopathy and indocyanine green angiography. Retina 1994;14:231-42.

17. Seong HK, Bae JH, Kim ES, et al. Intravitreal bevacizumab to treat acute central serous chorioretinopathy: short-term effect. Ophthalmologica 2009;223:343-7. 